\title{
A Novel WiMAX Structure with Mesh Network
}

\author{
Jie Zeng and XiaoFeng Zhong
}

\author{
Department of Electronic Engineering, Tsinghua University, Beijing, P.R. China 100084 \\ zengjie516@163.com
}

\begin{abstract}
IEEE802.16 (WiMAX) is a wireless metropolitan area network standard with high transmission speed and great coverage. Point to multi-point (PMP) is the traditional WiMAX transmission mode. Wireless mesh network (WMN) is an attractive and useful structure which is suggested to be adopted in WiMAX. A smart WiMAX structure with mesh is proposed in our paper, which supports direct data transmission between SSs (subscribe stations) with the control of the BS if possible. This mesh model is easy to achieve, and could improve the system performance such as throughput and propagation delay. With the help of the BS, several key problems in pure wireless mesh network are satisfactorily solved, such as coexistence, interference and billing. Simulation results are presented in this article to show the enhancement gain of the system performance by the smart model. The idea of virtual cell is put forward to benefit analysis, and the performance with AMC scheme is considered too. More concrete descriptions and explanations are given in the article.
\end{abstract}

Keywords: IEEE 802.16, WiMAX, wireless mesh network (WMN), adaptive modulation code (AMC), throughput.

\section{Introduction}

WiMAX (Worldwide Interoperability for Microwave Access) based on IEEE 802.16 standards is a wireless metropolitan area network (WMAN) which supports point to multi-point broadband wireless access(BWA). The IEEE wireless standard has a range of up to 30 miles, and offers wireless access at lower cost with higher bandwidth.It can afford transmission speed of up to 75 megabits per second. Therefore, WiMAX is considered one of the most attractive technologies for last-mile BWA problem in metropolitan areas and underserved rural areas. Mobile WiMAX based on the 802.16e-2005 enables WiMAX systems to address portable and mobile applications in addition to fixed and nomadic applications, and it seeks to support most client mobile requirements in the future market. The Mobile WiMAX Air Interface adopts Orthogonal Frequency Division Multiple Access (OFDMA) for improved multi-path performance in non-line-of-sight environments, Scalable OFDMA (SOFDMA)is introduced in the IEEE 802.16e Amendment to support scalable channel bandwidths from 1.25 to $20 \mathrm{MHz}$. Mobility broadens the market prospects of WiMAX, and creates a new research focus. 
Wireless mesh network, also known as ad hoc network, has become a significant technique for the next generation wireless networks. WMN is a self-organizing multi-hop system formed by user nodes,it can connect and communicate with other user nodes directly without pre-existing infrastructure. WMN provides a reliable and flexible system that can be quick-and-easy extended to thousands of devices. The signature of WMN is that each node acts as a relayed point for other nodes instead of being organized by a centralized control device. It has the reconstructive ability to fit the change of environments and the failure of some nodes. In addition, the distance of one-hop is much shorter; the power for transmission can be reduced obviously. Since the nodes in the multi-hop networking use much lower power to communicate with the neighboring nodes, the radio interference between them could be cut down. The channel quality and channel utilization efficiency will be greatly enhanced, resulting in higher capacity and better performance of the whole network.

WMN has so many advantages that it has done a good job in the improvement of IEEE802.11 network, known as the WiFi mesh. Similarly, it has been proposed to be adopted in WiMAX. To establish the multi-hop WMN connection and the single-hop PMP operation, IEEE 802.16a standard defined the basic signaling flows and message formats. The Mesh mode specifications are integrated into IEEE 802.16 revisions which are published later such as IEEE 802.16d and IEEE 802.16e.

The following is the key advantages of WiMAX mesh mode:

1) Greater coverage and better support of Non-Line of Sight (NLoS) transmission

2) Lower path loss and better performance

3) Higher throughput and lower delay

4) Rapid deployment and easy installation

5) Better robustness and flexibility

The name and the description of MAC Management messages for mesh network are given in IEEE802.16 standard, further work needs to be carried out to customize the specific application.

\section{System Model}

In order to achieve links characterized by high performances, the IEEE Standard 802.16a defines two different air-interfaces: PMP and Mesh. In PMP mode, connections are established among the BS and SS, hence, data transmissions between two SSs are routed through the BS. Within the Mesh network, traffic can occur directly among SSs since the protocol permits to set up data connections among neighbors. A station in the wireless network terms Mesh BS, acts like a BS in PMP mode and interfaces the network to the backhaul links. The MAC protocol for the Mesh mode has been designed to support both centralized (Mesh CS) and distributed (Mesh DS) schedulings.

A novel mesh structure is proposed as an extension to the traditional PMP WiMAX network, as is shown in fig. 1. When two subscribe stations (SS) are neighboring each other, they could exchange data packets directly with higher data rate and lower power after establishing the mesh link by the base station (BS) in the cell. 


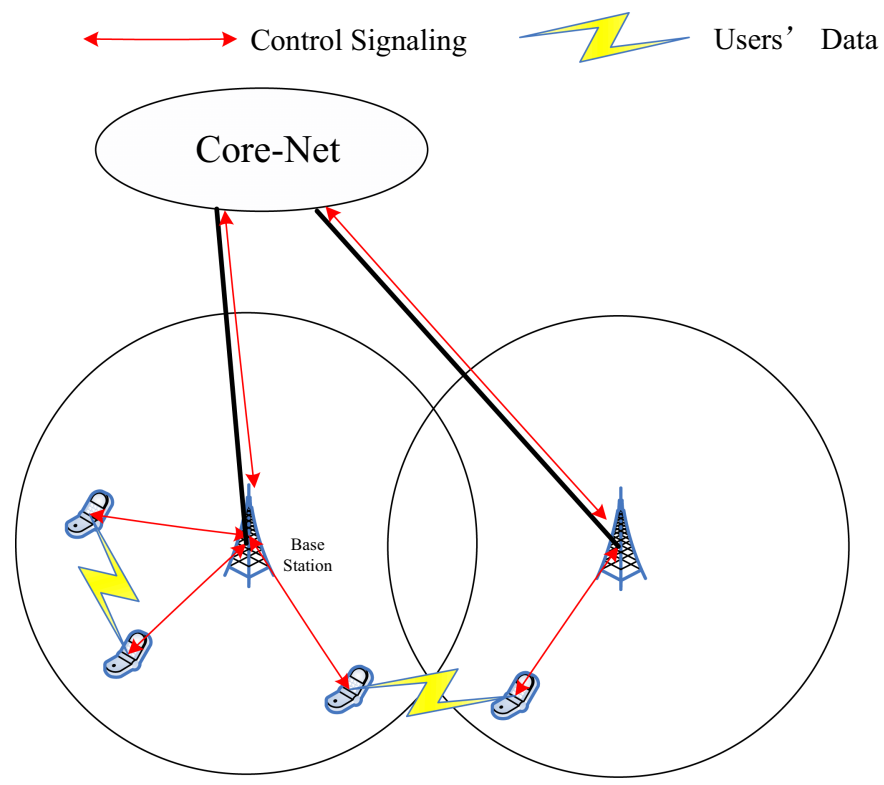

Fig. 1. Direct data transmission between SSs

Obviously, the scheduling idea we propose is simple to achieve and has a great system throughput enhancement. Through the control of a base station, cooperation shortcomings brought by DS mesh could be overcome, and the channel resources could be utilized more effectively. As a communication transfer and control center, the BS plays a very important role-key to the overall optimization. The main consideration in this issue is the system performance optimization within the coverage of one base station cell, and the cooperation between BSs to establish a mesh link needs pending further study.

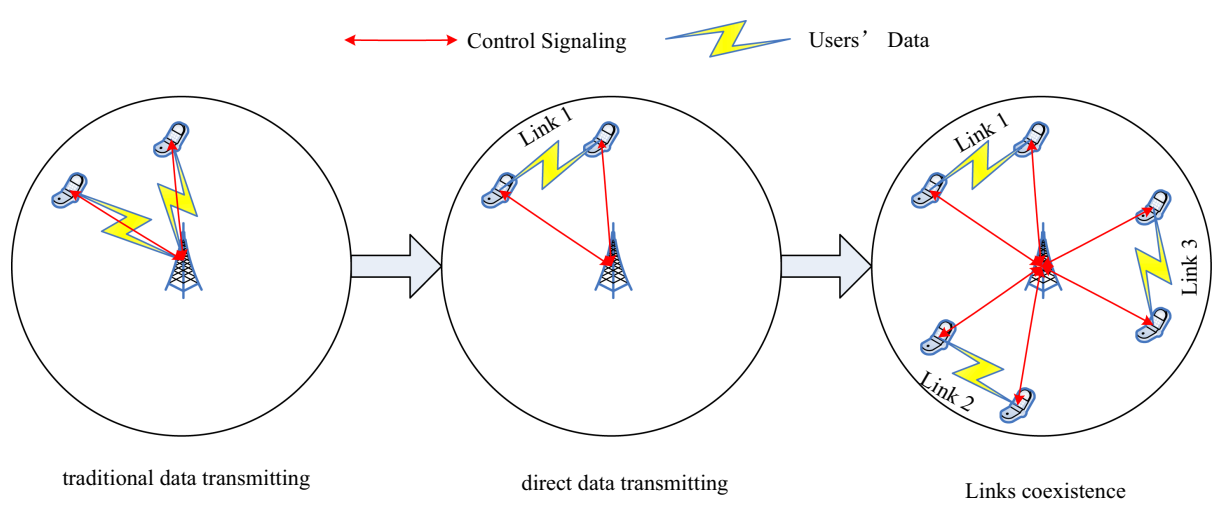

Fig. 2. Direct data transmission between SSs in one cell and different transmitting modes 
The scheduling mode can be simplified as is shown in the following fig. 2 when only one cellular area is considered. Within the scope of a cell, we allow more than one direct data transmission between SSs at the same time on condition that there is no interference. The system throughput could be much more improved since more than one mesh links work at one time.

Basic conclusions need to be given here preparing for the in-depth discussions afterwards.

Due to the difference of transceiver parameters(such as Transmitting power, Receiver sensitivity, antenna height and gain) between BS and SS, the coverage of SS to SS link is much shorter than that of SS to BS at the same bit rate. We assume that the radius of the cell is $R$, the distance between a pair of SSs is $d$, and a threshold for mesh communicating is D. Without considering the adaptive modulation control, a pair of SSs will communicate directly if and only if $d$ is not larger than D as proposed, otherwise they will transmit data with conventional mode through the BS.

If we put a pair of SSs in the cell randomly, fig. 3 gives the Cumulative Distribution curve of $d / R$.

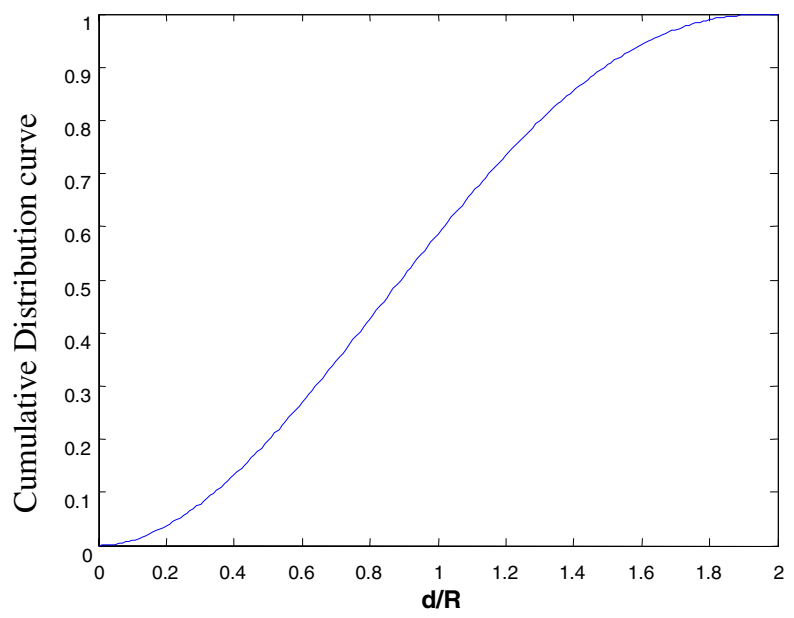

Fig. 3. The Cumulative Distribution curve of $d / R$ for a random pair of SSs

The transmission range between SSs is smaller than that between BS and SS according to the difference of transmission power, receiver sensitivity and the channel conditions (LoS or NLoS). Giving an estimate on the value of D/R makes sense, and enables a better discussion on this network structure. WINNER Work Package 5 (WP5) [1] refers to the multi-dimensional radio channel modeling, when the scenarios suburban macro-cell and urban macro-cell defined in the document is suitable for WiMAX. The WINNER channel models show the key features(such as large-scale parameters, antenna gain, path-loss models and probability of LoS) in wireless communication. The typical urban macro-cell model is chosen for an estimate on the 
value of D/R. The path-loss models have the form as in formula (1), and "dis" is the distance between transmitter and receiver:

$$
\mathrm{P}_{\text {loss }}=35.0 \log _{10}(\operatorname{dis}[\mathrm{m}])+38.4 \quad 50 \mathrm{~m}<\operatorname{dis}<5 \mathrm{~km}
$$

Generally, the base station transmitter power (TPBS) is from $30 \mathrm{dBw}$ to more than $43 \mathrm{dBw}$, and we choose $38 \mathrm{dBw}$ as the typical value. The transmitting power of the SS (TPSS) is from less than $10 \mathrm{dBw}$ to $33 \mathrm{dBw}$ (excessive estimate), and $28 \mathrm{dBw}$ is chosen as the typical value. Since we assume that the reception terminals and the channel path-loss models are the same, the transmission distance is just determined by the transmitting power at the same bit rates. According to the discussion, formula (1) comes up to formula (2) as following:

$$
\mathrm{TP}_{\mathrm{SS}}-\mathrm{TP}_{\mathrm{BS}}=35.0 \log _{10}(D / R)
$$

Using the assumed transmitter power of $\mathrm{BS}$ and SS, the typical D/R can be calculated 0.52. The more reasonable range of $\mathrm{D} / \mathrm{R}$ can be assumed from 0.3 to 0.7 . Basing on the proposed scheduling idea, the average throughput will be increasing while the value of $\mathrm{D} / \mathrm{R}$ increase.

A strongpoint in the model proposed is that there will be several direct non-interference transmission links in a cell when the threshold for mesh communicating D is suitable. A strongpoint in the model proposed is that there will be several non-interference direct transmission links in a cell when D is suitable. The coexisting restrictions of the direct transmission links should be discussed, since the scenario will bring more systematic throughput enhancements.

In the pure wireless mesh network, there are many solutions and schemes suggested to deal with the coexistence of several direct transmission links [2] [3]. A two-link coexisting condition will be discussed here to seek the detailed restriction shown in fig. 4.

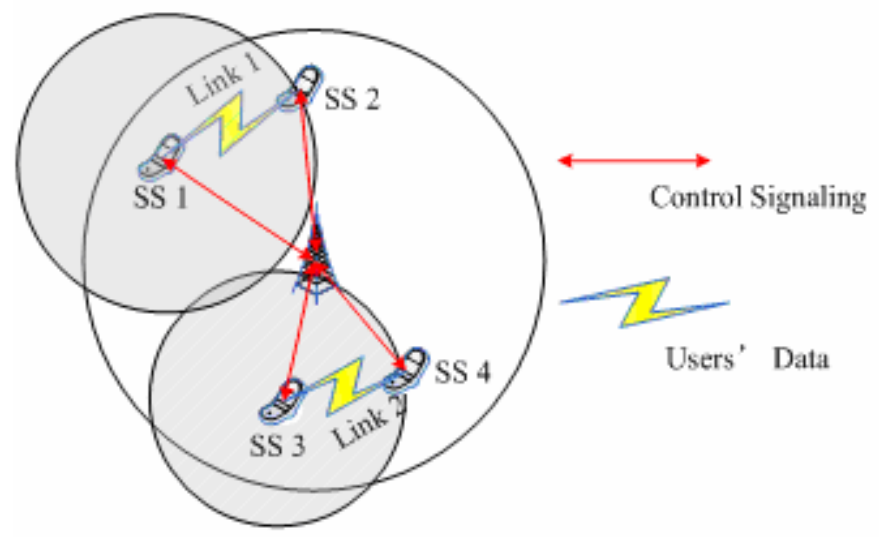

two coexistence links

Fig. 4. Two coexistence links scenario 
Basing on the power control, the adaptive modulation and coding (AMC) scheme adopted in the direct data transmission, virtual cells can be used to describe the scope of different bit rates and interference as shown in fig. 5 .

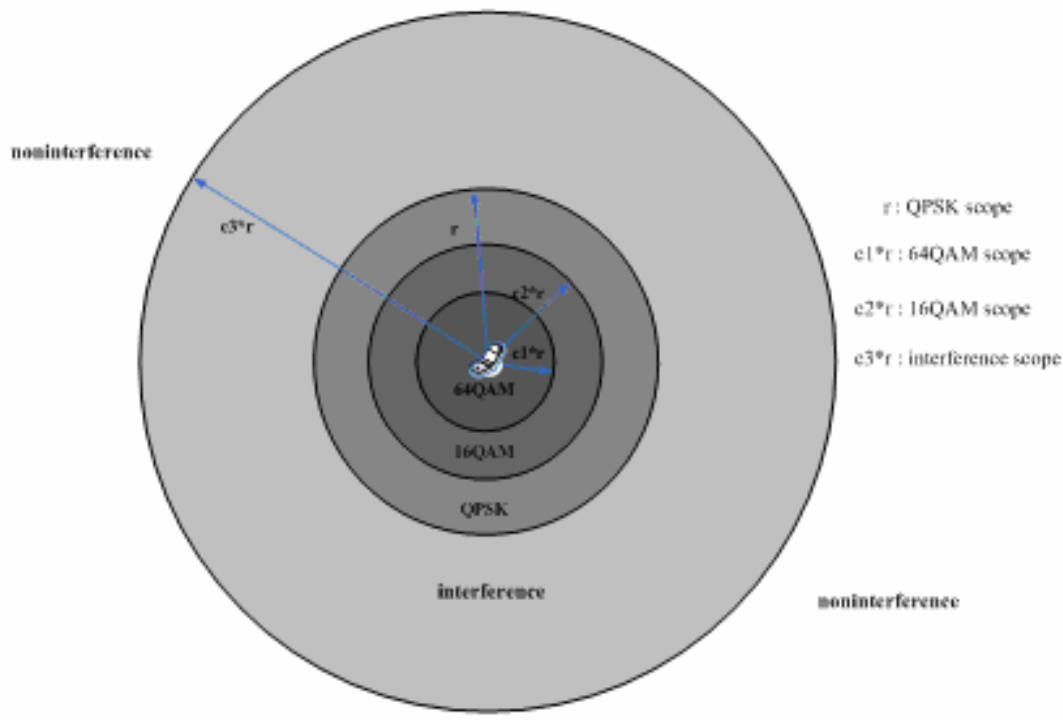

Fig. 5. Scope of different bit rates and interference

The virtual cell defined here is different from the traditional cell divided by the coverage of the BS, and is customized by the transmitter (BS or SS) launch capability. Since higher-order modulation means higher received power at the same BER (Bit Error Rate), there is smaller transmission scope according to the transmitting capability limit. As shown in fig. 5, different modulation modes have different transmission scopes. The typical modulation modes are QPSK, 16QAM and 16QAM. Several parameters are used to describe the application range. We assume that the QPSK transmission scope is $r$, the 64QAM scope is $\mathrm{c} 1 * \mathrm{r}$, and the 16QAM scope is $\mathrm{c} 2 * \mathrm{r}$. These adjustable parameters are set by the different wireless communication systems, and the typical value is given as follows: $\mathrm{r}=1000 \mathrm{~m}, \mathrm{c} 1=0.3, \mathrm{c} 2=0.6$. Interference between different transmission links is also considered, and virtual cell is also adopted to described the interference range $\mathrm{c} 3 * \mathrm{r}$. According to the large-scale transmission path-loss model, the signal to interference ratio (SIR) is more than $9 \mathrm{~dB}$ if the jamming 2 times the effective transmission distance away. In this condition the two links will coexist with the help of power control scheme as mentioned above, and the typical value of $\mathrm{c} 3$ will be $2 \sim 3$.

Essentially, the above-mentioned parameters are affected by the parameter $\mathrm{r}$ and three adjustable factors: c1, c2, c3. For different transmission equipments as BS and SS, the factors will be different as a result of the different equipment performances, transmissions and channel models. The factors are inconstant due to the various wireless 
communication systems. However, the three factors are of universal significance, and they will be determined when the equipment's performance is confirmed.

Two link coexisting can be regarded as this concretion: SS1 sends message to SS2 and SS3 sends message to SS4 simultaniously. The restrictions are: SS2 is in the effective transmission distance of SS1, SS2 is out of the interference scope of SS3; SS4 is the same as SS2 simply exchanging the positions of SS1 and SS3. The restrictions can be more intelligible and calculable by virtue of distance express as the following formula:

$$
\begin{aligned}
& \mathrm{d}(\mathrm{SS} 1, \mathrm{SS} 2)<=\mathrm{r}, \mathrm{d}(\mathrm{SS} 1, \mathrm{SS} 4)>=\mathrm{c} 3 \bullet \mathrm{r}, \\
& \mathrm{d}(\mathrm{SS} 3, \mathrm{SS} 4)<=\mathrm{r}, \mathrm{d}(\mathrm{SS} 3, \mathrm{SS} 2)>=\mathrm{c} 3 \bullet \mathrm{r}
\end{aligned}
$$

The coexistence restrictions of several links are in accordance with the previous conclusions, which makes sense in the coming simulation works. Take three-link coexistence as an example, the restrictions are:

$$
\begin{aligned}
& \mathrm{d}(\mathrm{SS} 1, \mathrm{SS} 2)<=\mathrm{r}, \mathrm{d}(\mathrm{SS} 3, \mathrm{SS} 2)>=\mathrm{c} 3^{\bullet} \mathrm{r}, \mathrm{d}(\mathrm{SS} 5, \mathrm{SS} 2)>=\mathrm{c} 3^{\bullet} \mathrm{r} \\
& \mathrm{d}(\mathrm{SS} 3, \mathrm{SS} 4)<=\mathrm{r}, \mathrm{d}(\mathrm{SS} 1, \mathrm{SS} 4)>=\mathrm{c} 3^{\bullet} \mathrm{r}, \mathrm{d}(\mathrm{SS} 5, \mathrm{SS} 4)>=\mathrm{c} 3 \bullet \mathrm{r} \\
& \mathrm{d}(\mathrm{SS} 5, \mathrm{SS} 6)<=\mathrm{r}, \mathrm{d}(\mathrm{SS} 1, \mathrm{SS} 6)>=\mathrm{c} 3^{\bullet} \mathrm{r}, \mathrm{d}(\mathrm{SS} 3, \mathrm{SS} 6)>=\mathrm{c} 3 \bullet \mathrm{r}
\end{aligned}
$$

\section{Performance Evaluation}

In this section we analyze the performance of the proposed network.

\subsection{Throughput Enhancement in Mesh Mode}

In the following simulations: $\mathrm{T}$ is the total simulation time. Tmax is the maximum traffic holding time, the actual traffic holding time is uniformly distributed from 0 to tmax. Num is the number of total traffic links. The throughput of traditional WiMAX cell is normalized as 1, and all the links take the same QPSK modulation. The following figs. 6, 7 and 8 give the simulation results of the mesh modes of WiMAX throughput enhancements.

The direct mesh communicating will achieve double throughput enhancement for a single link. Considering the SSs distance probability and threshold D, the average system throughput will be increased by nearly $20 \%$ when the value of $D / R$ is justifiably selected as 0.6 show in fig. 6 and 7. For different systems the threshold D can be changed, fig. 8 shows how the system throughput changes when the $D / R$ changes. Obviously, more direct communications link when $\mathrm{D} / \mathrm{R}$ is large,because this means more throughput enhancement, the simulation results verify the same conclusions. Fig. 9 gives a more clear relationship between throughput enhancement and the value of D/R. According to the curve and the analysis above, it is recommended to choose achievable largest threshold $\mathrm{D}$ in the system, such as 0.7 . 


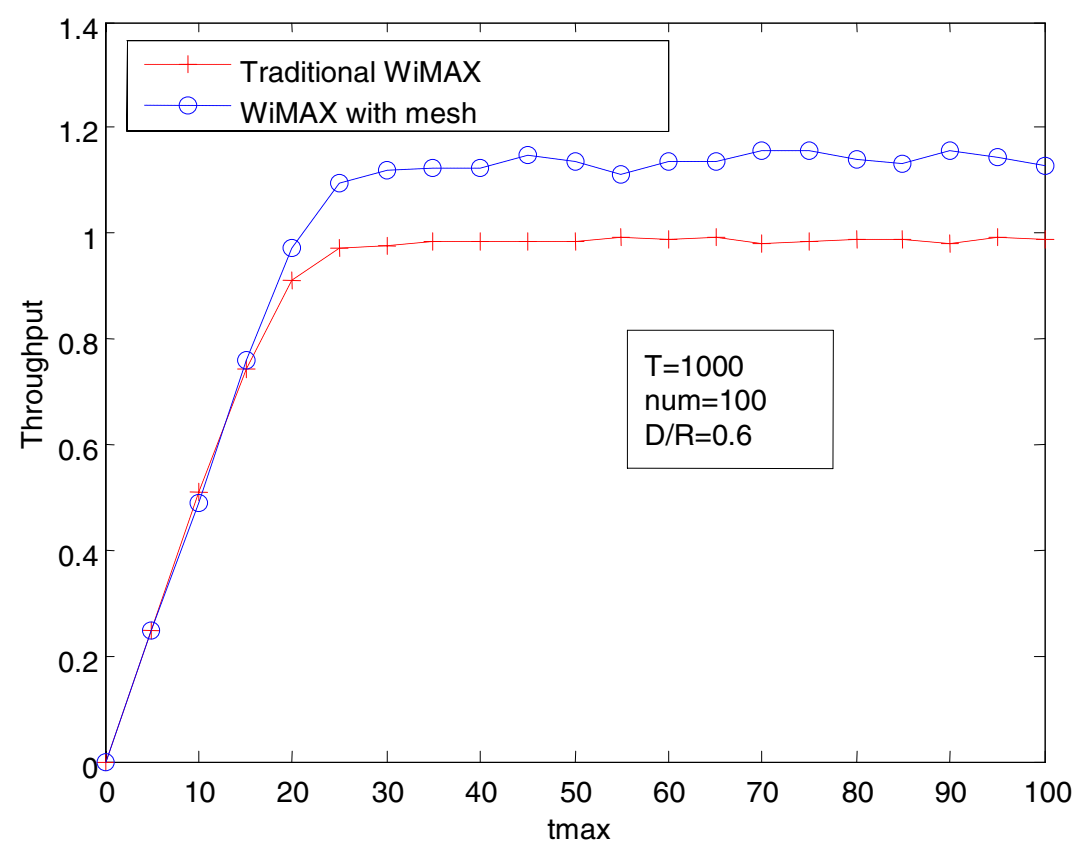

Fig. 6. System throughput under the changing of tmax

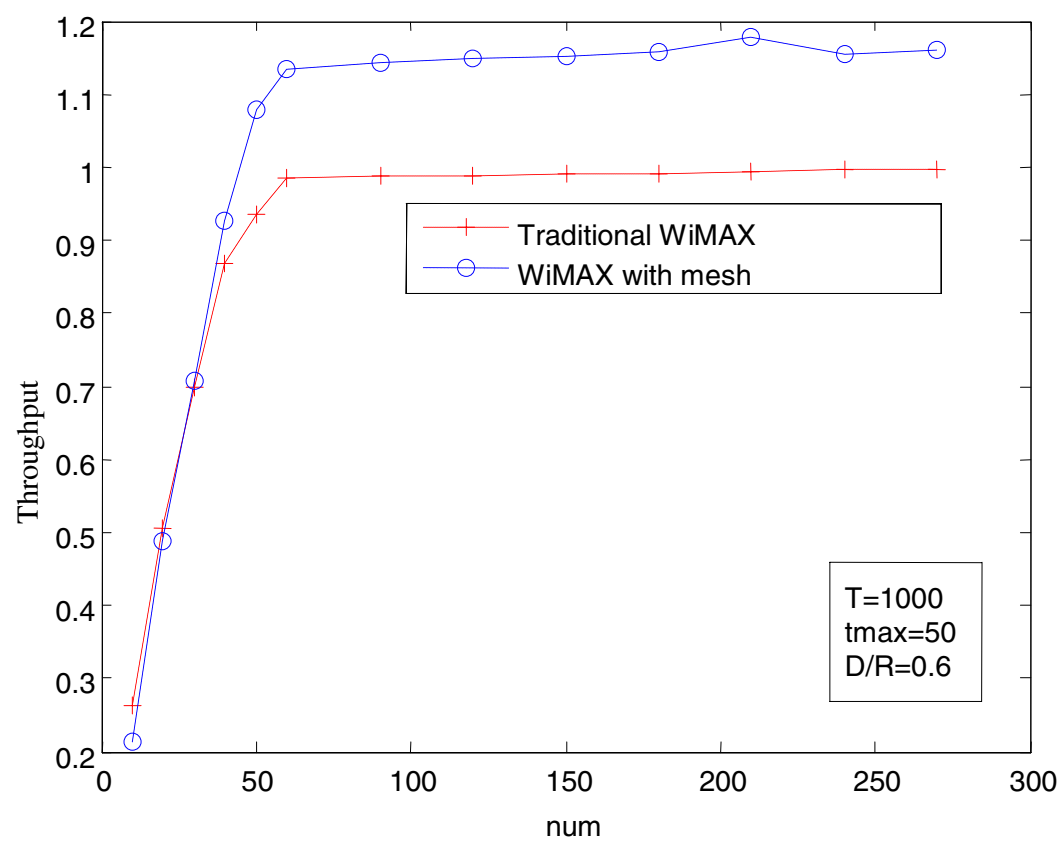

Fig. 7. System throughput under the changing of num 


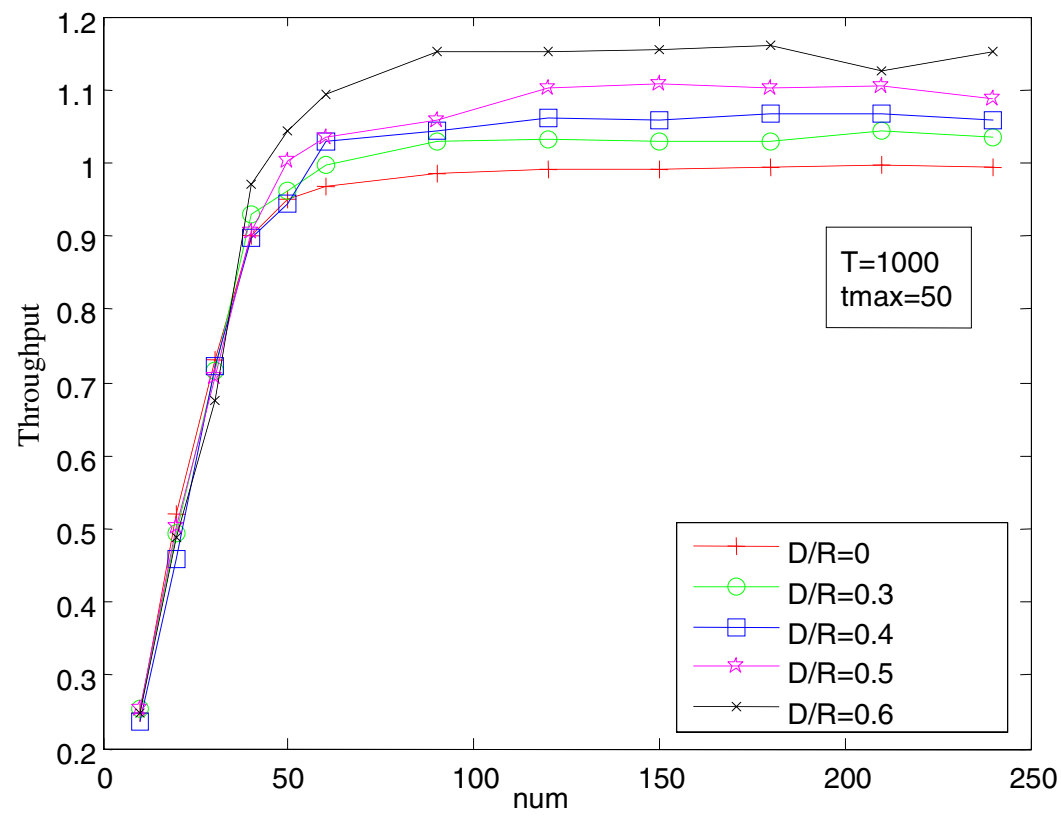

Fig. 8. System throughput under the changing of $D / R$

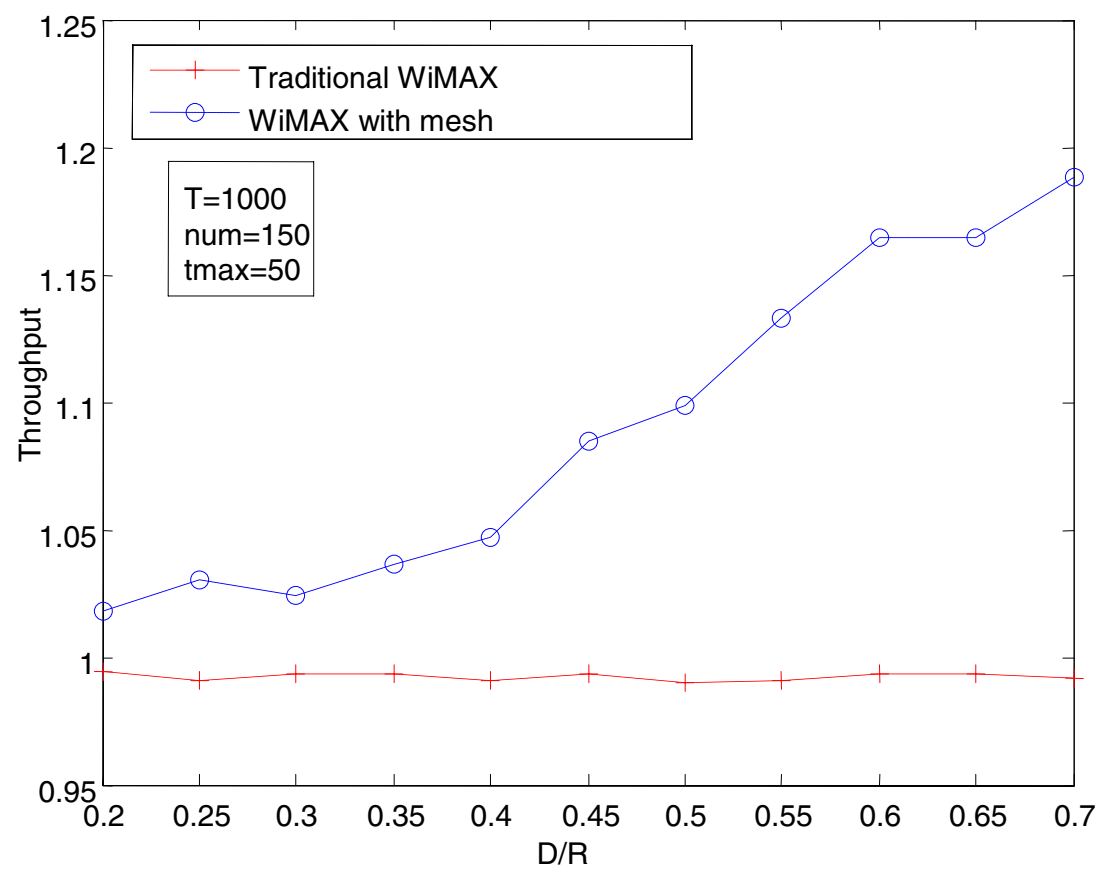

Fig. 9. System throughput under the changing of $D / R$ 


\subsection{Throughput Enhancement with AMC}

Throughput enhancement with AMC is discussed in the following simulation. With the adaptive modulation and coding scheme that are defined in IEEE802.16e specification, the neighboring SSs of shorter distance could achieve higher data rate, which will lead to more throughput enhancement. The parameters of the virtual cell need to be confirmed to support the simulation:

In the traditional WiMAX, the SS will work on different modulation ranks of 64QAM, 16QAM and QPSK when the distance between SS and BS is 0.3R, 0.6R and R. In the virtual cell of the $B S$, rate $1=\mathrm{c} 1=0.3$, rate $2=\mathrm{c} 2=0.6$.

In the WiMAX mesh mode, the SS will work on different modulation ranks of 64QAM, 16QAM and QPSK when the distance between a pair of SSs is $0.3 \mathrm{D}, 0.6 \mathrm{D}$ and D. In the virtual cell of the SS, rate $3=\mathrm{c} 1=0.3$, rate $4=\mathrm{c} 2=0.6$.

Rate1 4 are parameters in the simulation, they are set in a certain system. Fig. 10 gives the throughput comparison with AMC, which will be increased by nearly $40 \%$ in a cell while the AMC scheme gains $20 \%$ throughput enhancement.

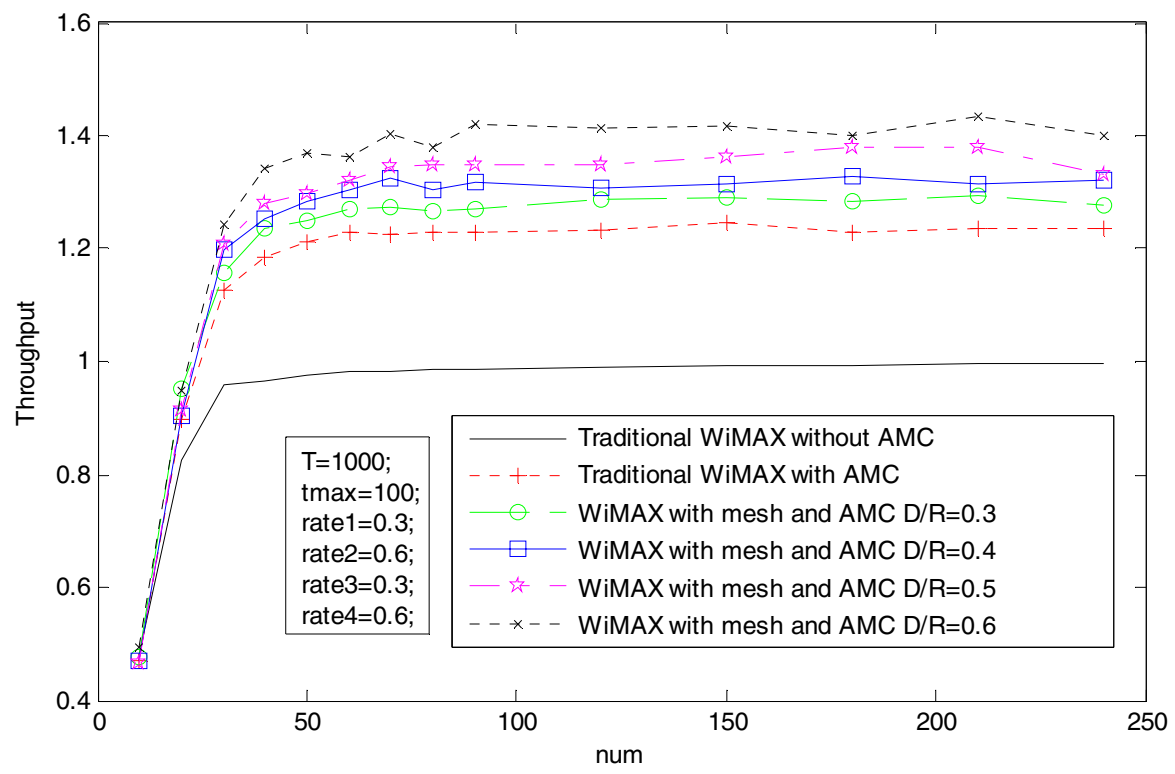

Fig. 10. Throughput comparison with AMC

The virtual cell parameters change in different systems, since the SSs are more likely to communicate in high modulation ranks, the virtual cell parameters of the SS may be set as rate $3=\mathrm{c} 1=0.6$, rate $4=\mathrm{c} 2=0.9$. This adjustment will bring changes in system performance as is shown in fig. 11, throughput enhancements therefore directly link and are more likely to work in high transmission speed. 


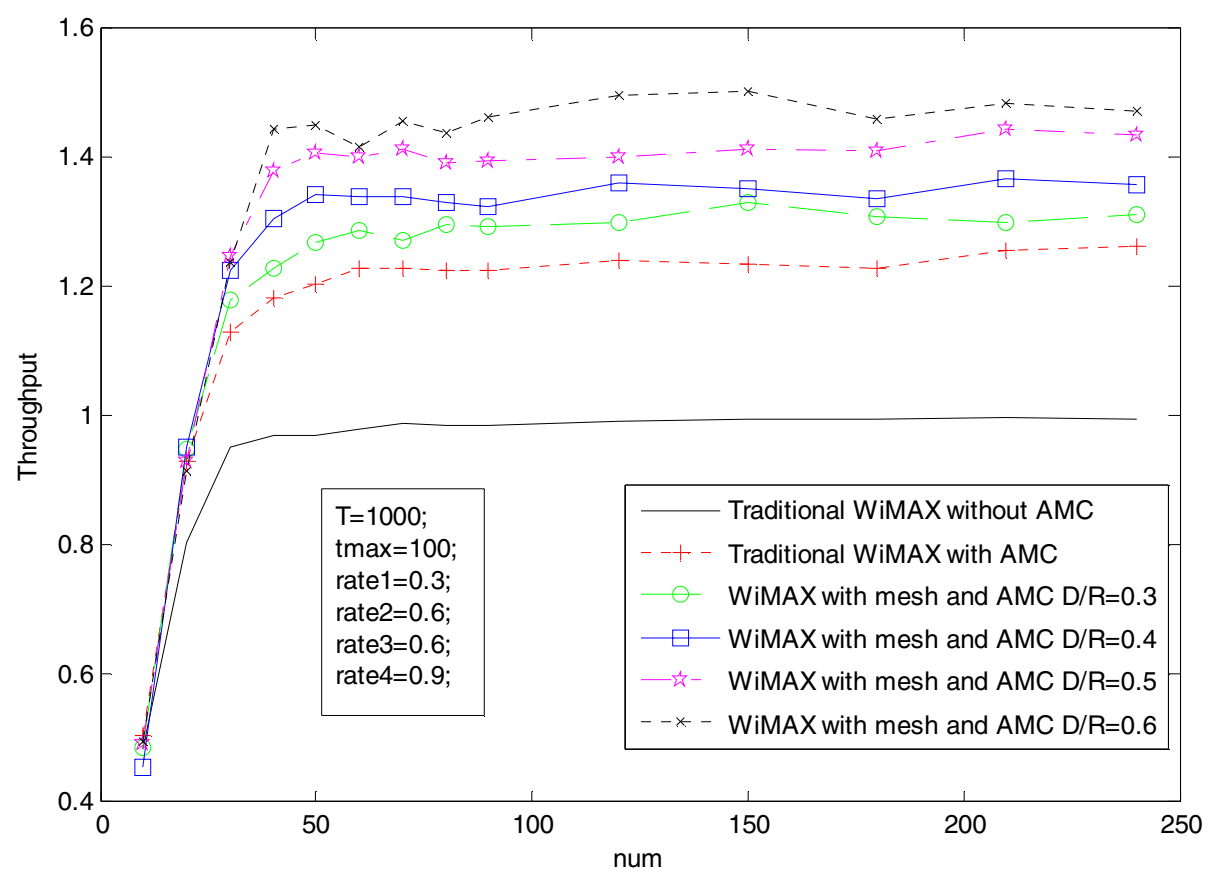

Fig. 11. Throughput comparison with AMC

\subsection{Performance Enhancement with Power Control to Enable Coexistence}

The system's overall performance increase is concerned with power control techniques, which enables the coexistence of direct transmission links. For link coexistence scenario, if the threshold for mesh communicating D decreases, there will be more direct links work in one channel. However, it can be seen from the cumulative distribution curve of $\mathrm{d} / \mathrm{R}$ that decreased $\mathrm{D}$ means the probability of direct link is decreasing. Fig. 12 shows that the probabilities of two direct links arrive at one time.

Since the probability of coexistence is very small, it has minimal impact on overall system throughput. But for a single communication link itself, this state makes sense. In uneven distribute business, or even artificially optimized wireless communication systems, coexistence scenario appears more frequently. It reflects the very good strength and directly enhances the system performance. The number of direct transmission links which can coexist in the given $\mathrm{D} / \mathrm{R}$ is present in Fig. 13 as a performance indicator.

It is very obvious that by support of the power control techniques, when D is decreased, more links can communicate simultaneously without interference in a cell. This coexistence scenario can be applied to some specific scenes which have frequent and close communication. For example, in one building, the main wireless business is the communication within the same office. 


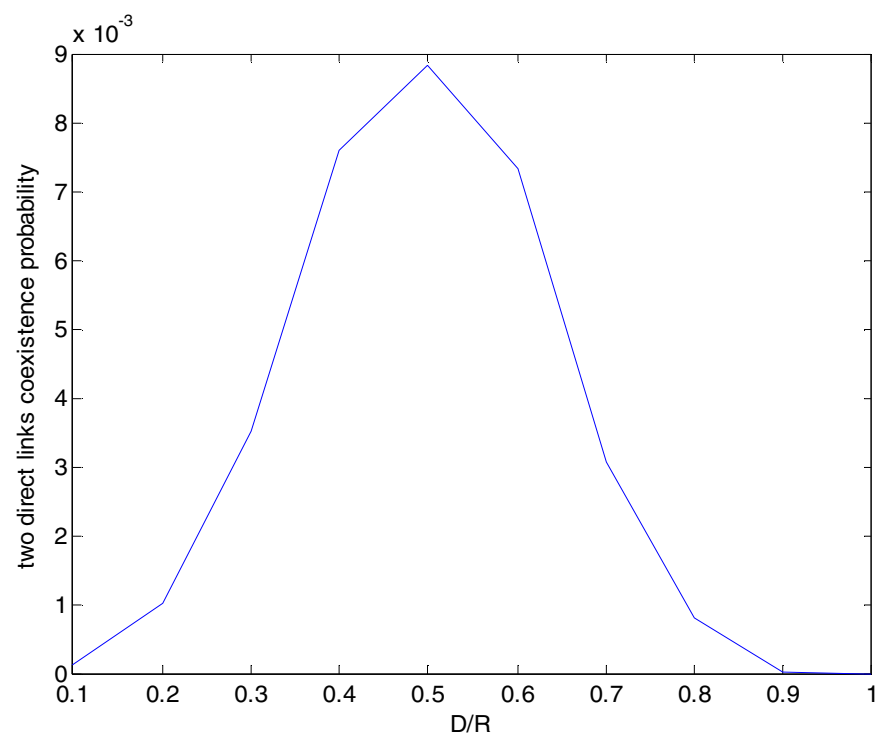

Fig. 12. Probabilities of two direct links arrive at one time

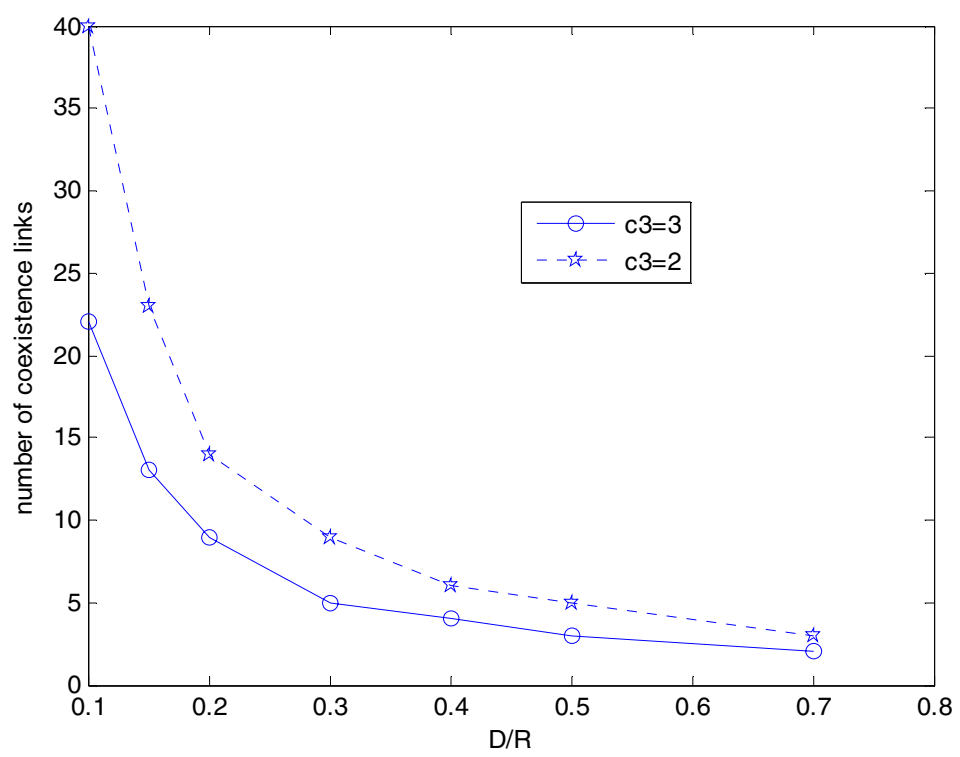

Fig. 13. The number of direct transmission links can coexist in given $D / R$

\section{Conclusion and Further Work}

The structure referred in the article is based on the mesh network under the control of the BS according to the characteristics of WiMAX. The greatest advantage of such a 
structure is the easiness of carrying out and taking substantial gains. The communications in the cell are scheduled by the BS; FIFO (First In First Out) mode is adopted to enable best delay performance. Compared with pure mesh network without the control of BS, the system resources are more effectively used, the coexisting restrictions and management problems are no more hard to deal with. For a single link, direct data transmission without being transmitted through BS means shorter transmission delay. Further more, the distance between SSs is suitable for high level modulation, which increases the transmission speed. The link throughput could enhance $2+$ times when the pair of SSs transmits data directly. When the threshold for mesh communicating D increases, more pairs of SSs could communicate directly, and this leads to more enhancements in system throughput. AMC technology proposed to be adopted in the WiMAX standards improves the system performance visibly, and it also plays a good role in the mesh model. The virtual cell is a good tool for the analysis and description of the AMC scope, and it also helps solving interference problems in the coexistence of several links.

Simulation results are given to confirm the architecture advantages; they also give constructive comments on the system parameters design. The enhanced designs for direct communication capabilities between SSs can support the proposed architecture, and it's the future trend of the development of distributed communication systems.

From the emergence of the concept, wireless mesh network has achieved good results in both theory and practice. However, as evolved over from the traditional computer networks, WMN has several inevitable shortages in telecommunication business. The structure proposed combines the advantages of WiMAX and WMN, and is very simple to achieve. The service quality is well defined in WiMAX, which brings reliable telecommunication applications. The transmission delay of individual business could be reduced using mesh, and the average system throughput also enhances much. The combination of WiMAX and WMN achieves remarkable improvements in system performance, too.

In this article, we focus on the network structure, the working model and system performances. In-depth research needs to be carried out in specific business QoS requirements and $\mathrm{BS}$ scheduling optimization. Simulation results in this article are based on one cell scope and paired communication business. FIFO is used as the business processing scheduling program, which can be considered as the most optimal manner of the single link latency. But FIFO may not be the best choice in times of transmission congestion. To seek the optimum of the overall throughput, some communication businesses need to be rejected and the coexisting of several links are desirable.

Mesh network under the control of BS is discussed in this article, which is also known as CS Mesh. From another perspective, DS Mesh means the coexistence of different links and restrictions of data transmission. The structure proposed requires corresponding changes when used in pure mesh or DS mesh network.

In all, our smart structure combines the advantages of two advanced technologies, and is highly feasible. The scheduling optimizing could be highly targeted to adapt well to the future telecommunications development. 


\section{References}

1. IST-2003-507581 WINNER D5.4 v. 1.4 Final Report on Link Level and System Level Channel Models

2. Whitehead, P.: Mesh Networks; a new Architecture for Broadband Wireless Access Systems. In: RAWCON (2000)

3. Redana, S., Lott, M., Capone, A.: Performance Evaluation of Point-to-Multi-Point (PMP) and Mesh Air-Interface in IEEE Standard 802.16a. In: Vehicular Technology Conference, 60th edn., pp. 3186-3190. IEEE, Los Alamitos (2004) 